\title{
Extraction and Usage of Starch from Banana Pseudostem to Develop Biodegradable Polymer Composites
}

\author{
Samarasekara A.M.P.B. , Chamikara A.W.C. and Wijesundara W.W.H.P. \\ Department of Materials Science and Engineering, University of Moratuwa, Katubedda, Sri Lanka \\ banduamp@yahoo.com
}

\begin{abstract}
Polymer products have become an integral part in our daily life as a basic need. It produced on a massive scale worldwide and its production crosses the 150 million tonnes per year globally. Polymers have broad range of applications in films, wrapping materials, shopping and garbage bags, fluid containers, clothing, toys, household and industrial products and building materials. Polymer materials play a dominate role in the packaging applications today. Most of packaging materials are based on petroleum based by-products. These products may remain for centuries in our surrounding environment after the usage causing lots of environmental issues. Biodegradable polymers are the one of the options available to protect the environment. Most biodegradable polymers can be engineered to be composted safely in a properly managed, municipal composting environment and breakdown occurs in a combination of water and microbial activity. Most of natural polymers are biodegradable in nature. Increasing interest in applying polymers based on natural materials such as starch has been observed. Starch is a bio-material and one of the most abundant and inexpensive polysaccharide sources which has the unique characteristic of biodegradability and easily dissolve in water. Blends of synthetic polymers and starches can be prepared so they are biodegradable. The objective of this research is to develop biodegradable composite materials using starch and Low Density Polyethylene (LDPE).
\end{abstract}

Banana is a very popular fruit in Sri Lanka. It is cultivating in most of the areas of the country. The banana pseudostem was selected as a fruit waste to extract starch. Starch was extracted from different varieties banana pseudostems such as Rathkesel, Puwalu, Suwadel, Ambul kesel, Alukesel and Ratahondral. The maximum starch amount (5 wt \%) was given by Puwalu pseudostem. Fourier Transform Infrared (FTIR) Spectroscopy was used to characterize the extracted starch. Experimental results showed that all characteristic peaks related to starch were present in the extracted FTIR spectra. FTIR spectra further showed that starch composition was almost same in all the banana plants. Laboratory scale internal mixer at $150{ }^{\circ} \mathrm{C}$ temperature and speed of $60 \mathrm{rpm}$ was used to prepare the LDPE - starch polymer mixture by varying $1 \mathrm{wt} \%$ to $5 \mathrm{wt} \%$ starch. LDPE - starch biodegradable polymer composite was prepared with the help of a hydraulic press. Tensile properties, soil burial test and water absorption tests were carried out to measure the degradability of the developed product. There was a significant weight lost in starch containing composite samples during the soil burial test. Tensile test results were also showed that considerable property changes in starch containing samples with compared to pure LDPE. LDPE - starch composites are exciting and useful materials to protect the environment.

Keywords: Starch, LDPE, Biodegradable, Banana Pseudostem 